doi.org/10.51891/rease.v7i6.1443

\title{
DISCALCULIA: E SUA RELAÇÃO COM O CÉREBRO
}

\author{
Luciana Pereira Dourado ${ }^{1}$
}

RESUMO: O presente artigo apresenta um estudo acerca do funcionamento do cérebro e seu envolvimento no processo da aprendizagem a fim de compreender qual a sua relação com o distúrbio da discalculia, termo usado para se referir à falta de habilidade que alguns indivíduos apresentam em resolver problemas matemáticos que também é definido como um transtorno neuropsicológico que apresenta características como a dificuldade no processo de aprendizagem de calcular, ou falhas no raciocínio lógico matemático, identificada com o CID ı. Assim o objetivo deste ensaio é compreender o fenômeno da discalculia e sua relação com as áreas do cérebro, para sugerir formas de intervenção pedagógica. Esse assunto é de grande importância para a família, a comunidade escolar e a sociedade. Com o propósito de promover maior clareza este artigo apresenta algumas áreas cerebrais por meio de ilustrações, e descrição de cada uma com enfoque naquelas envolvidas com a resolução de problemas matemáticos. Com a intenção se chegar a um resultado satisfatório, optou-se pela pesquisa bibliográfica, qualitativa, utilizando a entrevista semi estruturada que serviu para a coleta de dados apresentados, bem como, a realização de observações durante a pesquisa, e aplicação de teste neuropsicológico infantil conhecido como bateria Luria desenvolvido para identificação e diagnóstico da discalculia. Para a realização do estudo e melhor compreensão da temática, apoiou-se nos estudos de diversos autores que discorrem sobre a discalculia, como Flávia Heloísa dos Santos, Jussara Bernardi e Ana Maria Antunes de Campos estes renomados autores e pesquisadores vem trazendo em suas obras não somente um, mas os diversos tipos de discalculia. Dessa forma este estudo leva a conclusão do quanto se faz necessário aprofundar em pesquisas acerca da discalculia buscando condições de ajudar aqueles que a possuem.

Palavras-chave: Discalculia. Funcionamento do Cérebro. Papel docente frente à discalculia.

ABSTRACT: This article aims to present a study about the functioning of the brain and its involvement in the learning process, in order to understand what is its relationship with dyscalculia disorder, a matter of great importance for the family, the school community and society, a Since the disapproval and disgust in relation to the discipline of mathematics is something already rooted in history, to understand the reasons why this disturbance related to mathematics occurs, it is necessary to understand how the brain works, and how it behaves in the face of learning situations , from the individual's birth to his maturation and what are the processes involved in the acquisition of knowledge, as well as the processing of information and which the triggered areas suffer failures or have been injured so that the disorder under study occurs For a better understanding the article has been bringing some figures. With the intention of reaching a search result, I seek $\mathrm{u}$ study through different authors who talk

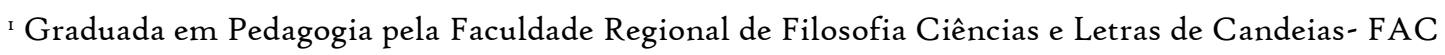


about dyscalculia, which despite being still a topic with little scientific interest already arouses the curious eye of renowned authors and researchers that has brought in their works not only one, but the different types of dyscalculia. We will also present the results and conclusions of the neuropsychological test for children and semistructured interviews with the family of a child applied to a child with suspected dyscalculia. And we end this article with suggestions on how teachers should deal with these children and how to help them move forward from the disorder.

Keywords: Dyscalculia. Brain Functioning. Teaching role in the face of dyscalculia.

\section{INTRODUÇÃO}

As habilidades desenvolvidas pela disciplina da matemática como resolver problemas adotando diferentes estratégias, somar, subtrair, dividir, raciocinar de maneira lógica, entre outras, estão presentes em grande parte das atividades desenvolvidas no nosso cotidiano, por isso o domínio de tais habilidades é de grande importância tanto na vida escolar quanto na vida social. Sem contar que elas estão ligadas diretamente ao desenvolvimento da autonomia e do senso crítico do sujeito.

Entretanto, é cada vez mais notável o quanto os alunos apresentam uma resistência a disciplina de matemática, além de apresentarem cada vez mais baixo desempenho.

Em consonância com os resultados apresentados pelo PISA e Prova Brasil realizado com alunos do ensino fundamental, médio e graduação no ano de 2013, a matemática se tornou uma das disciplinas que mais reprovam os alunos desde o ensino fundamental até o ensino médio.

Por isso é necessário que educadores e pesquisadores busquem compreender os fatores que desencadeiam o baixo desempenho destes estudantes, uma vez que este fator pode estar relacionado a problemas neurológicos e internos do desenvolvimento do sujeito frente à disciplina.

Assim o objetivo deste ensaio é compreender o fenômeno da discalculia e sua relação com as áreas do cérebro, para sugerir formas de intervenção pedagógica.

$\mathrm{O}$ artigo vem trazendo o resultado de algumas pesquisas a respeito do tema discalculia, onde se optou pela pesquisa bibliográfica, que também é classificada qualitativa, sendo utilizado como instrumentos para investigação de pesquisa, a entrevista semi estruturada que serviu para coleta de dados. 
Dessa forma a pesquisa apresenta, inicialmente, a relação entre: A aprendizagem e o cérebro: definindo o conceito de aprendizagem e como esse processo está presente na vida humana desde o seu nascimento, assim como o comportamento do cérebro humano diante de situações de aprendizagem, com foco nas áreas cerebrais acionadas na aprendizagem da matemática. Para tanto procurou-se averiguar e conceituar a Discalculia: Uma desordem no cérebro trazendo o conceito de discalculia, seus diversos tipos e causas. Por fim as intervenções que devem ser feitas diante do diagnóstico.

Ao finalizar este ensaio, conclui-se o quanto é necessário que professores e pesquisadores busquem aprofundar-se nos estudos acerca da discalculia, um distúrbio que atinge crianças e adolescentes muito mais comuns do que se imagina levando os na maioria das vezes a desistir de sua vida escolar.

\section{A APRENDIZAGEM E O CÉREBRO}

É cada vez mais difícil encontrar uma definição que consiga abranger de forma completa todo o processo de aprendizagem, pois não se pode defini-lo apenas como a entrada e saída de informações, nem somente considerá-lo a partir de sua área emocional. O processo de aprender envolve aspectos cerebrais, psíquicos, cognitivo e social. Assim podemos dizer que é um processo neuropsicocognitivo que ocorrerá em um determinado momento, em uma determinada sociedade, ou ainda dentro de uma cultura em particular. De acordo PIMENTEL; LARA (2015)

\footnotetext{
Desde o início do século XXI, sabe-se conforme Rodrigues e Novaes (2014) que o cérebro humano possui como principal característica a capacidade de adequar novas informações em seu sistema à medida que conhece e/ou estuda pessoas novas e coisas novas. Essa característica dá-se o nome de plasticidade, justificada pelo modo como o cérebro é capaz de introduzir novos dados aos já existentes. Como resultado dessa plasticidade cerebral, obtêm-se a aprendizagem, caracterizada pelo modo como as pessoas apreendem coisas novas, acrescentando-as ao cérebro, mantendo-o ativo, com novas informações.
}

É válido lembrar que a bagagem adquirida com as experiências passadas, sentimentos, vivências e situações sociais influenciam diretamente sobre o aprendizado. É através de nossa estrutura psíquica que conseguimos dar significados aos processos perceptivos, enquanto a organização cognitiva sistematiza as 
informações que recebemos de maneira particular, segundo as experiências e as situações em que ocorreram por isso os sujeitos da aprendizagem e suas maneiras de aprender são resultados de práticas sociais e culturais.

A aprendizagem não pode de maneira alguma ser considerada uma ação passiva de recepção de informação, nem tão pouco isolada, pelo contrário, atualmente tem se falado muito em aprendizagem interativa, da dimensionalidade do saber, como sendo algo que supõe uma construção individual do sujeito que, para na sua aquisição utiliza de todos os recursos oferecidos a seu favor, essa construção ocorre de maneira interna e subjetiva, processada e com interação.

Desde a gestação que o ser humano estar envolvido no processo de aprendizagem, pois é através dele que consegue atingir a maturidade e o desenvolvimento como indivíduos num mundo que exige o tempo todo interação com o meio, assim é possível concluir que a aprendizagem ocorre durante toda a vida humana, que a mesma é um processo integral que ocorre desde o princípio da vida.

Toda vez que aprendemos algo novo o nosso cérebro sofre modificações tanto físicas, quanto químicas, para compreender seu desenvolvimento e funcionamento é preciso conhecer a anatomia e fisiologia do sistema nervoso que é dividido em duas partes: sendo elas o sistema nervoso central e o sistema nervoso periférico, conforme mostra a figura abaixo:

\section{FIGURA I}

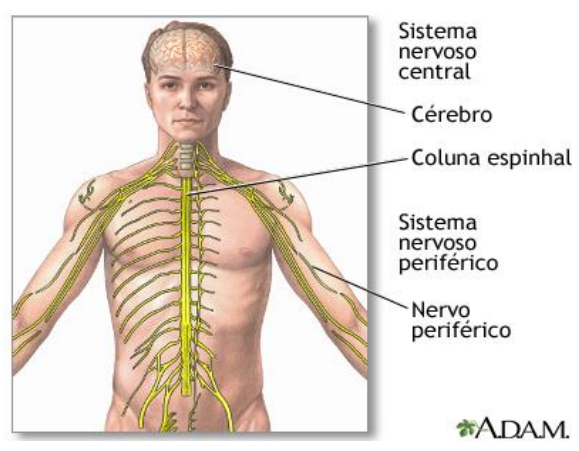

https://ssl.adam.com/graphics/images/pt/8679.jpg

O sistema nervoso central é formado pelo cérebro, cerebelo e a medula espinal. O cérebro fica localizado no final da coluna vertebral e estar dividido em duas 
importantes regiões, os hemisférios direito e esquerdo que por sua vez contam com quatro lobos: frontal, parietal, temporal e occipital. A união desses dois hemisférios se dá através do corpo caloso. Já o cerebelo se encontra abaixo do lobo occipital e é responsável por funções como o equilíbrio, a coordenação motora, além de servir de conexão entre a medula e o cérebro. Pesquisas comprovam sua importância na coordenação dos pensamentos emoções e memórias e sentidos durante o processo cognitivo. De acordo Luria (1981,p.27)apud Bernardi

[...] uma estrutura hierarquizada que consiste em pelo menos três zonas corticais construídas uma acima da outra: as áreas primárias que recebem impulsos da periferia ou os enviam para ela;as secundárias ,onde as informações que chagam são processadas e, finalmente as terciárias, os últimos sistemas dos hemisférios cerebrais a se desenvolverem e responsáveis, no homem, pelas formas mais complexas de atividade mental que requerem a participação em concerto de muitas áreas corticais.

Assim segundo Luria o homem ao realizar ações como fala, uso da memória, pensamento e fala, utiliza um sistema altamente complexo.

E por fim a medula espinal que é formada por células nervosas responsáveis pela transmissão de informações e mensagens por todo o corpo. $O$ sistema nervoso periférico é dividido em somático e autônomo e é responsável pelas funções vitais do nosso corpo.

Para compreender a relação entre a aprendizagem e o cérebro buscaremos aprofundamento acerca do sistema límbico que está localizado sobre o bulbo raquidiano e que tem suas estruturas duplicadas nos dois hemisférios cerebrais, é ele que controla diversas funções entre elas podemos citar: a alimentação, a reprodução e a geração de emoções, além disso, é dividido em quatro partes e todas estão relacionadas ao processo de aprendizagem. (FIGURA 2):

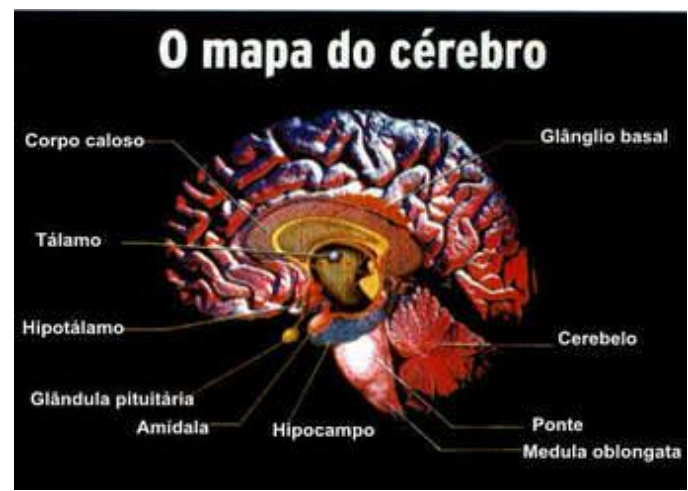

https://media4.picsearch.com/is?HYdPa2a3iu25DQwmDhEOErX_27GyoliTUA,E5EorCJ8\&height 
Conforme pensamento de Bernardi

O fato da participação grupal das estruturas cerebrais operarem conjuntamente explica por que as funções mentais não podem estar localizadas em áreas restritas do córtex ou em agrupamentos celulares isolados. Assim justifica o fato de ser considerado todo o sistema nervoso central

É possível concluir que o cérebro funciona em conjunto e por se apresentar como uma função integrada de todo o sistema nervoso central, em que devem participar diferentes regiões as funções de cognição precisam estar íntegras.

Dando seguimento teremos o tálamo que é o principal receptor de informação sensorial, o hipotálamo que entre outras funções vitais é também o responsável por algumas funções psíquicas e psicomotoras, o hipocampo que é o principal quando se trata de aprendizagem uma vez que é ele que transforma informações a partir da memória e por meios de sinais elétricos e as envia para as regiões de armazenamento de longo prazo, levando para isso o tempo entre alguns dias ou até meses. O hipocampo está sempre em processo de revisão das informações relacionadas à memória de curto prazo fazendo comparações com as lembranças das experiências armazenadas, dando enfim, sentido a cada uma delas. E por último nem por isso menos importante a amígdala que se encontra colada ao hipocampo e desempenha papel fundamental em relação às emoções, principalmente o medo, é possível, embora ainda não comprovado que ela seja a responsável pelo armazenamento do conteúdo emocional das lembranças e dos componentes cognitivos.

O contato com nova situação de aprendizagem provoca uma série de mudanças no cérebro, causadas pelas sinapses, que podem ser definidas como a comunicação entre os neurônios células nervosas cerebrais. Assim toda vez que o ser humano é desafiado a resolver uma situação problema ele realiza novas conexões dando continuidade à formação da rede de neurônios que permite ao cérebro nova organização.De acordo (Gomez;Terán, 2014. p.39)

a conexão que existe entre uma célula e outra por onde viajam as mensagens por meio de substâncias químicas chamadas de neurotransmissores. Desse modo a informação é transferida de uma célula a outra. As sinapses ocorrem principalmente...

a célula nervosa recebe informação de outra célula nervosa ou de órgãos sensoriais e projeta essa informação para outros neurônios .Esses neurônios projetam essa mensagem para todos as partes do corpo 
Essa rede de neurônios é flexível com capacidade de melhorar-se e adaptarse a qualquer situação.

Ainda de acordo com Gomez e Terán o cérebro humano estar sempre se reorganizado formado novas conexões. Essas mudanças são o produto da maturação e das experiências, também se sabem que as diferentes partes do cérebro apresentam momentos diferentes para aprender, são as chamadas janelas de oportunidade que podem ser definidos como importantes períodos nos quais o cérebro responde a certos estímulos ou consolida conexões nervosas, esses estímulos podem vir principalmente do ambiente.

\section{I.I- Processamento de informações pelo cérebro}

Outro fator importante para esta pesquisa é conhecer a maneira como o cérebro realiza o processamento das informações, como são selecionadas aquelas que serão armazenadas na memória de longo prazo e as que logo em seguida serão descartadas. Assim neste capítulo vamos no deter no estudo da forma como coletamos, avaliamos guardamos e recuperamos as informações. Este processo se da em três importantes etapas: registro sensorial, memória de curto prazo e memória de longo prazo.

Registro ou memória sensorial, este sistema envolve o tálamo e o sistema reticular, e é nesta etapa que as informações são captadas ao nosso redor, os estímulos ingressam em nosso cérebro através dos impulsos elétricos que são produto da conexão de rede de neurônios, porém é necessário que haja uma seleção, pois se o cérebro tivesse que registrar todas as informações recolhidas pelos sentidos entraria em pane, pois de acordo com Gomez e Terán (2014)estima-se que um cérebro de um adulto consegue guardar na faixa de sete pacotes de informações, enquanto o cérebro infantil apenas três.

Essa seleção ocorre com base na importância que a informação captada tem para o indivíduo, é o que denominamos de registro sensorial.

Após ser selecionada no registro sensorial, a informação é então lançada à memória de curto prazo, que está dividida em dois estágios: a memória imediata que como o próprio nome sugere, retém a informação por um período de tempo muito 
curto, cerca de 30 segundos aproximadamente e seu processamento ocorre tanto subconscientemente quanto conscientemente. O outro estágio é chamado de memória de trabalho, neste caso o processamento se dá de maneira consciente, ou seja, quando armazenamos alguma informação na memória de trabalho é sinal que a mesma possui certo nível de importância, e é a partir deste estágio que somos capazes de separar, reunir e refletir sobre as informações decidindo armazená-las em outro lugar ou não.

Com base nos estudos de Gomez e Terán (2014) a memória de trabalho é também temporária, possuindo um limite de 15 a 25 minutos para que a informação seja armazenada permanentemente na memória de longo prazo, onde permanecerá para sempre e poderá ser resgatada sempre que houver necessidade, ou ser descartada para sempre. Para realizar o processo de seleção de informações ela utiliza alguns critérios, quando nos defrontamos com uma situação de aprendizagem alguns critérios são fundamentais, um deles é o sentido, ou seja, precisamos compreendê-lo a partir de nossas experiências para depois retê-lo. Outro critério é o significado, o material somente será retido se tiver relevância. Em ambos os casos é válido ressaltar que o fator primordial que dá sentido e significado as informações são as experiências do sujeito.

E por fim teremos a memória de longo prazo, sua capacidade de armazenamento é praticamente ilimitada, seu funcionamento é dinâmico e interativo que cataloga e arquiva as informações em grupos, ou níveis diferentes que sofrem mudanças e reorganização toda vez que outra informação é selecionada. É esta organização que permite a recuperação das informações todas as vezes que precisamos.

A memória de longo prazo é subdividida em diferentes módulos: memória declarativa e a processual. A declarativa é onde guardamos todas as informações objetivas como datas, nomes, números, e que está subdividida em memória semântica onde estão os fatos relacionados a vivência de mundo, as regras da lógica, graças a ela é que conseguimos nos lembrar da tabuada por exemplo. E a memória episódica responsável por guardar nossos detalhes biográficos.

Já a memória processual é responsável pelas nossas habilidades e hábitos. 


\section{0: DISCALCULIA: UMA DESORDEM NO CÉREBRO}

O termo discalculia é usado para se referir à falta de habilidade que alguns indivíduos apresentam em resolver problemas matemáticos. É definido como um transtorno neuropsicológico que apresenta características como a dificuldade no processo de aprendizagem de calcular, ou falhas no raciocínio lógico matemático, identificada com o CID ro.

Segundo o DSM (Manual Diagnóstico e Estatístico de Transtornos Mentais: DSM-V/(American Psychiatric Association):

[...] discalculia é um termo alternativo usado em referência a um padrão de dificuldades caracterizado por problemas no processamento de informações numéricas, aprendizagem de fatos aritméticos e realização de cálculos precisos ou fluentes. Se o termo discalculia for usado para especificar esse padrão particular de dificuldades matemáticas, é importante também especificar quaisquer dificuldades adicionais que estejam presentes, tais como dificuldades no raciocínio matemático ou na precisão na leitura de palavras. (DSM Apud PIMENTEL Letícia da Silva; LARA Maria Cristina Machado de.2015).

Assim podemos concluir que a discalculia pode ser considerada como uma desordem estrutural da maturação das capacidades matemáticas, sem, porém apresentar quaisquer desordens em outras áreas da aprendizagem, mas não se pode desconsiderar que a mesma esteja associada a outro distúrbio como, por exemplo, a dislexia, sendo a falta de compreensão da leitura ou até mesmo a incapacidade de realizá-la, as razões da dificuldade no trato com a matemática. Nesta perspectiva poderia ser definida como um processo de falta de maturação cerebral, mas não lesional.

Segundo classificação feita por Kosc, ela pode ser classificada em três tipos:

I:Discalculia verbal:onde a dificuldade consiste em apresentar dificuldade em nomear quantidades matemáticas,os números,termos e símbolos.

2:Discalculia practognóstica:dificuldades em enumerar, manipular objetos reais ou em imagens e comparar.

3:Discalculia léxica :dificuldades na leitura dos símbolos matemáticas

4:Discalculia gráfica: dificuldades na grafia de símbolos matemáticos

5:Discalculia ideognóstica: falta de capacidade para realizar operações de cálculo mental e compreensão dos símbolos matemáticos. 
6:Discalculia operacional: incapacidade de realização de operações e cálculos numéricos.

Além desses seis tipos classificados, Campos (2015) em suas pesquisas, afirma que a discalculia se divide em três classes:

I - Natural: ocorre quando a criança apresenta dificuldades pelo fato de não ter tido contato com o processo de contagem, então não terá conhecimentos suficientes para compreender o raciocínio matemático.

$2^{-}$- Verdadeira: mesmo após sofrer várias intervenções o sujeito não apresenta modificações favoráveis ao seu raciocínio lógico matemático.

$3^{\text {a }}$ Secundária : a dificuldade em aprender matemática pode esta associada a outros distúrbios como a dislexia,conforme citado anteriormente.

Assim torna se essencial, que estes sintomas ao serem observados pelo professor, o mesmo possa alertar a família para a busca de profissional habilitado para ajudar o estudante discálculio através de atividades psicopedagógicas.

O processo de calcular é uma atividade bastante complexa, mesmo em um cálculo simples envolve vários processos cognitivos, segundo Bernardi (2014,p.25)

Por ser um funcionamento cognitivo uma função integrada do sistema nervoso central, em que devem participar diferentes regiões corticais, tornase possível concluir que as funções cognitivas necessitam estar íntegras e em pleno funcionamento para que a aprendizagem aconteça, pois as mesmas constituem-se em condições básicas para que ela se processe. De qualquer forma uma alteração da capacidade de cálculo e do manejo de números, cálculo mental, leitura e escrita dos números - caracterizando uma discalculia, é manifestada em diferentes regiões do cérebro pelas especialidades diferenciadas que cada hemisfério desempenha.

Em consonância com o pensamento de Bernardi pode se chegar à conclusão de cérebro funciona em conjunto, ou seja, para realizar uma ação várias estruturas são acionadas, quando ocorre deficiência em qualquer uma dessas áreas tem consequência a deficiência da ação, ela não terá o sucesso esperado. As habilidades matemáticas segundo visão de Piaget são construídas a partir de funções como a memória de longo e curto prazo, raciocínio e orientação espacial, porém estudos atuais revelam que já nascemos com áreas cerebrais específicas para o aprendizado da matemática, o que leva a crer que qualquer dano, trauma ou imaturidade levem ao desenvolvimento da discalculia. Ainda segundo Bastos (2016) O'Hare e outros colaboradores descreveram dois diferentes quadros clínicos que estão ligados ao hemisfério prejudicado, se o 
hemisfério direito foi comprometido caracteriza a inabilidade em conceituar quantidades numéricas, porém sem prejudicar o reconhecimento e a produção simbólica de números, enquanto o comprometimento do hemisfério esquerdo estaria relacionado a falta de habilidade em reconhecer, produzir números e símbolos operacionais, neste caso o conceito de quantidade numérica não seria prejudicado, mas outras habilidades como cálculo mental, montagem de sequências numéricas, memória auditiva de curto prazo, estariam prejudicadas.

Chegamos assim à conclusão, de que diferente de alguns autores que consideravam apenas a deficiência no hemisfério esquerdo, ambos estão envolvidos no processo do desenvolvimento matemático, sendo a discalculia a consequência de uma desordem cerebral em qualquer hemisfério tanto o direito quanto esquerdo, uma vez que os procedimentos utilizados na matemática ocorrem tanto em um quanto em outro.

\subsection{O PAPEL DO PROFESSOR DIANTE DO ALUNO DISCÁLCULO}

Ao se notar sinais de discalculia o professor deve aconselhar os pais a buscarem profissionais habilitados para que possam realizar testes que confirmam ou não a suspeita de discalculia, ela pode ser diagnosticada através de testes psicopedagógicos e exames de imagem, os testes ajudaram identificar os sintomas apresentados pelos discálculios.

Os sintomas apresentados são: Erro na formação de números, que geralmente ficam invertidos, como se fosse uma imagem em espelho; Dislexia; Inabilidade para efetuar somas simples; Inabilidade para reconhecer sinais operacionais e para usar separações lineares; Dificuldade para ler corretamente o valor de números com multidígitos; memória fraca para fatos numéricos básicos; dificuldade de transportar números para local adequado na realização de cálculos; ordenação e espaçamento inapropriado dos números em multiplicações e divisões.

Assim que o diagnóstico for feito a intervenção deve ocorrer imediatamente e ser focada no aspecto aritmético propriamente dito, ou seja, as remediações devem se relacionar aos conhecimentos aritméticos básicos, ações como redução do tempo de respostas em tarefas de contagem de itens não simbólicos, permitir a ampliação de 
precisão na estimativa de números em linha numérica entre outras podem ajudar o aluno a vencer as barreiras no aprendizado da matemática.

A discalculia pode ser diagnosticada através de testes psicopedagógicos e exames de imagem, os testes ajudam identificar os sintomas apresentados pelos discálculios.

É importante que o professor busque através da auto formação maneiras de intervir de forma a ajudar seu aluno a sair dessa zona de desconforto, buscar atividades que proporcione a eles interação, participação e socialização desenvolvendo assim o raciocínio lógico, a adoção de metodologias que favorecem a utilização de materiais concretos, uso de calculadoras. É valido ressaltar que alunos discálculios têm sim condições de aprender basta que o professor esteja disposto a não economizar nos recursos de apoio. Também é importante investir em atividades lúdicas e outras que ajudem a elevar a autoestima do aluno e auto-imagem, pois geralmente estes indivíduos apresentam uma imagem negativa de se mesmo e se sentem diferentes pelo fato de não aprenderem da maneira e no ritmo dos outros.

\section{4.o APRESENTAÇÃO E DISCUSSÃO DE DADOS}

O teste neuropsicológico foi aplicado a pedido da professora que notou desenvolvimento tardio do aluno na área de matemática, em relação aos demais colegas. Ela relata que a criança sente muita dificuldade na realização das atividades de matemática, e ainda necessita muito de apoio material concreto, e muitas intervenções observou também que o mesmo não consegue realizar cálculos mentais simples, realizando contagem com auxílio dos dedos no caso de operações como adição e multiplicação, há relato também do fato dele não conseguir memorizar fórmulas ou cálculos simples. Essas queixas despertaram na equipe escolar um olhar diferenciado e investigativo acerca desse aluno que já conta com io anos de idade e frequenta o quarto ano do ensino fundamental de uma escola particular.

Em entrevista com os pais do aluno, foi possível tomar conhecimento de fatos importantes de seus primeiros anos de vida que podem estar relacionados ao seu desenvolvimento aritmético, a mãe relatou que sua gravidez foi de alto risco devido à ocorrência de pré- eclampsia, conforme pesquisa de Bernardi (2014) a aprendizagem é 
um processo que se inicia no sujeito antes mesmo do seu nascimento e só dissemina com a morte. O que nos leva a refletir acerca desses acontecimentos presentes desde a vida intra-uterina desse sujeito. O tipo de parto foi normal, apesar dos problemas durante a gravidez e parto, ela conseguiu amamentá-lo ate próximo três anos de idade, no primeiro ano de vida iniciou o processo de andar e aos 2 anos a criança já conseguia controlar os esfíncteres porém em relação ao desenvolvimento da fala só iniciou após I ano e meio de idade com dificuldades que prevalecem até os dias atuais (Io anos), sua dicção não é clara e ocorre troca de letras de som semelhantes tanto na fala quanto na escrita.ainda segundo Gomez e Terán (2015,p.140)

Da mesma forma que os transtornos fonéticos, todos os outros assinalados são encontrados nas crianças, neste caso até aproximadamente até os.Se persistirem após essa idade é um chamado de atenção que deve ser considerado. [...].

Diante dessa observação, surge uma nova suspeita de que, o aluno pesquisado seja portador de outro distúrbio que leve a discalculia, em todo caso não se pode desconsiderar nenhuma hipótese de investigação.

Durante entrevista a mãe do aluno pesquisado relatou que ele sofreu queda com forte pancada na cabeça quando começou a frequentar a escola, um colega o empurrou no portão no momento da saída, além do mais, sua infância foi marcada por diversos problemas de saúde principalmente no trato respiratório e infecções das vias urinárias, problemas esses que foram a razão da evasão escolar desse aluno no primeiro ano escolar.

A mãe relatou como é seu comportamento no âmbito familiar onde afirma que ele é uma criança calma, gosta de ajudar, é ativo e comprometido, mas apresenta resistência na realização das tarefas de matemática, este relato é bastante semelhante ao feito pela professora quando questionada sobre o comportamento do aluno na sala de aula. A mãe também relata a necessidade de atividades lúdicas para vencer a resistência dele na realização das tarefas de matemática. Uma ação que tem mudado aos poucos o comportamento do mesmo. De acordo Campos (2015, p.57)

Pesquisas comprovam que podemos e devemos ensinar matemática usando como instrumento de aprendizagem a atividade lúdica. A criança se torna mais livre e mais social desenvolvendo a sua capacidade visual, auditiva, 
tátil e conceitual. A ludicidade estimula o raciocínio lógico, o pensamento independente, a criatividade e a capacidade de resolver problemas.

Assim conclui-se da importância de buscar alternativas que aumentem a motivação da criança em relação a sua aprendizagem, quando o aluno se torna motivado consequentemente também se torna participativo, pensante e independente.

Durante entrevista também foi possível descobrir que tanto o pai quanto a mãe da criança apresentaram dificuldades de aprendizagem no início da vida escolar, a mãe teve bastante dificuldade na aquisição de leitura e escrita, e seu pai um processo semelhante ao da criança em relação aos conhecimentos matemáticos, e ainda hoje na vida adulta tem dificuldade de concentração.

O resultado do exame de autoestima e autoimagem revelou que a criança possui uma autoimagem negativa quando responde que se pudesse mudaria algumas coisas do seu passado, deseja ter uma memória melhor e não se declara como uma criança realizada na escola. Gostaria às vezes de ter uma vida diferente, e admira a vida de outras crianças em detrimento da sua. Miras Apud Bernardi esclarece:

A maioria das aproximações atuais de análise da dimensão emocional e afetiva dos processos educacionais escolares compartilha alguns pressupostos básicos, algumas noções e alguns conceitos que evidenciam pontos de contato e possibilidades de integração de suas respectivas colaborações.

Entre ele, destacam os conceitos vinculados as representações que construímos de nós mesmos e sobre os demais

Neste fragmento fica evidente a importância dessas dimensões no processo de aprendizagem, que se inicia no sujeito antes mesmo do seu nascimento e só dissemina com a morte. O que nos leva a refletir acerca desses acontecimentos presentes desde a vida intra-uterina desse sujeito

Ela mesma declarou ser uma criança medrosa destacando o medo da morte, que possivelmente pode estar relacionado com o momento da gravidez e parto que se apresentou como um momento muito tenso e que as emoções de alguma forma o tenham afetado mesmo sem que a mãe percebesse efeitos imediatos.

A realização do teste neuropsicológico ou bateria Luria como também é conhecido, segundo afirma Bernardi (2014) é um teste criado com base em $\mathrm{O}$ Diagnóstico Neuropsicológico de Luria para adultos, instituído por Manga e ramos em 1991. Durante a realização do teste foi possível observar que a criança foi realizando 
alguns com muita facilidade, em relação aos números de ordens de dezenas e centenas simples, na leitura e escrita, nas operações aritméticas o mesmo conseguiu realizar com contagem nos dedos, porém o teste permitia que ele pudesse usar papel na realização desta tarefa, quando usou o papel para uso do algoritmo convencional o mesmo se atrapalha quanto a posição dos números, confirmando o fato relatado anteriormente sobre a necessidade de suporte concreto e intervenções, ao solicitar que o mesmo realizasse a contagem dos números de trás para frente a partir do número ıoo, a criança declarou que não conseguia, mas fez com alguns números abaixo de 30. Também foi observado durante o teste que ele apresenta dificuldades em acessar dados na memória de trabalho, como no caso de cálculos simples. De acordo SANTOS (2017,p. 62)

\begin{abstract}
Kucian e von Aster (2015) elencam as queixas que essencialmente caracterizam a discalculia: prejuízo do senso numérico; dificuldades para estimar quantidades, reduzida capacidade de subitização; dificuldade com a transcodificação de representações simbólicas; dificuldades para contar em ordem inversa; incompreensão do sistema decimal; prejuízo no desenvolvimento da linha numérica mental; capacidade limitada de recuperação de fatos numéricos; dificuldades para decompor um problema em partes; incompreensão dos procedimentos de cálculo e seus conceitos
\end{abstract}

O aluno apresenta, ora sim ora não características que poderiam classificá-lo em algum dos tipos de discalculia

Ao término dos testes muitas especulações ainda permanecem pelo fato de reconhecermos que para um diagnóstico seguro seria necessária a realização de outros testes para verificação de outros possíveis transtornos, como uma dislexia leve, ou outros fatores como a falta de maturidade, amadurecimento tardio, ou ainda um déficit entre idade cronológica e idade mental. Dessa forma a mãe foi aconselhada a buscar apoio psicopedagógico, psicológico, fonoaudiólogo para que possam ser realizados outros testes que possam levar a conclusão do que acontece de fato com o aluno.

\title{
CONSIDERAÇÕES FINAIS
}

A partir desta pesquisa e de tudo que foi observado e exposto, fica clara a importância de se dar atenção a crianças que ficam nervosas, passam mal e que se recusam a participarem das aulas de matemáticas, segundo o que conseguiu averiguar a discalculia se apresentam em diversas formas e tipos por isso muitas vezes relato de 
desinteresse e preguiça pode representar um transtorno de aprendizagem contra o qual o aluno não conseguirá vencer sem ajuda adequada.

Também podemos perceber que o diagnóstico não é algo que se obtém com facilidade devido a tantos fatores a serem considerados, assim como os seus diversos tipos. Sendo necessária a utilização de testes desenvolvidos especialmente para diagnosticá-la, e esse processo de diagnóstico envolve vários profissionais.

Por isso são importantes estudos que levem a compreensão ampla da discalculia, a fim de encontrar formas de ajudar crianças portadoras do distúrbio a se superarem e ter uma vida escolar digna, uma vez que exceto a discalculia verdadeira, os outros tipos podem ter seus quadros amenizados ou até mesmo erradicados. Apesar de recentes e de pouco interesses científicos as pesquisas sobre transtorno de discalculia já são um subsídio muito importante para que o discálculio possa ter garantido o seu direito de aprender defendido por lei.

Uma criança com discalculia é um sujeito que precisa com urgência ser resgatada em todas as dimensões da vida: emocional, orgânica e social, além da dimensão intelectual, não se deve ignorar que ela tem no seu histórico de vida o fracasso, humilhações e frustrações por não conseguir aprender, e se ela não aprende a responsabilidade não deve ser atribuída somente a ela com certeza há parcelas que devem ser distribuídas entre família escola e a sociedade.

Por isso é importante um olhar diferenciado para esses indivíduos e enxergálos além do que se podem ver superficialmente, suas dificuldades e também suas potencialidades, respeitando lhes as limitações buscando sanar suas necessidades. As instituições de educação precisam se preparar como um espaço configurado para atender a seus alunos em todas as suas necessidades educativas e potencializar o seu desenvolvimento integral impedindo a repetência, o fracasso, a evasão e em consequência a exclusão social.

\section{REFERÊNCIAS BIBLIOGRÁFICAS}

BASTOS, José Alexandre. Transtornos da aprendizagem : abordagem neurobiológica

e Multidisciplinar [recurso eletrônico] / Organizadores, Newra Tellechea Rotta, Lygia Ohlweiler, Rudimar dos Santos Riesgo. -2. ed. - Porto Alegre : Artmed, 2016. 
BERNARDI, Jussara .Discalculia: o que é?Como intervir?.Paco editorial:Jundiaí 2014 CAMPOS,Ana Maria Antunes de.Discalculia superando as dificuldades em aprender matemática. Wack editora.rio de Janeiro.2015

CID-ı. Classificação de Transtornos Mentais e de Comportamento da CID-ı: Descrições clínicas e diretrizes diagnósticas. Organização Mundial de Saúde (Org.). Porto Alegre: Artes Médicas, 1993.

GIL, A. C. Como elaborar projetos de pesquisa. 4. ed. São Paulo: Atlas, 2007.

GÓMEZ.Ana Maria Salgado; TÉRAN.Nora Espínola. Dificuldades de aprendizagem.Cultural. S. A. Brasil,2014

GOLDENBERG, M. A arte de pesquisar. Rio de Janeiro: Record, 1997.

MINAYO, Maria Cecília de Souza (org.). Pesquisa Social. Teoria, método e criatividade. 18 ed. Petrópolis: Vozes, 2001

PIMENTEL, Letícia da Silva; Mestre em Educação em Ciências e Matemática /

PUCRS, Licenciada em Pedagogia, Pós-graduação em Supervisão Escolar. leticiapimentel_pedagogia@yahoo.com.br LARA, Maria Cristina Machado de Pós-doutorado em Educação em Ciências e Matemática pela PUCRS. Doutora e Mestre em Educação pela UFRGS. Licenciada em Matemática pela UFRGS. isabel.lara@pucrs.br.O cérebro e as Habilidades matemáticas. 